\title{
MICTURATING CYSTOURETHROGRAPHY AS A PART OF URINARY TRACT INFECTION WORK UP IN CHILDREN
}

\author{
Shrestha $\mathbf{S}^{*}$, Adhikari $\mathrm{N}^{*}$
}

\section{ABSTRACT}

Urinary tract infection (UTI) is a common infection in childhood and has the potential to cause renal damage especially in younger children ( $<2$ years). Almost 30- $40 \%$ cases of UTI are associated with vesicoureteral reflux (VUR). The main objective of this study was to identify the presence of VUR in children with UTI and its relative distribution according to age and sex. This is a retrospective hospital based study carried out between Jestha 059 to Poush 060 (15/6/2002-15/1/2004) at department of Paediatrics, Patan Hospital. All the cases that underwent micturating cystourethrography (MCUG) as part of UTI work up were included in the study and the information was collected from patient record files. During this period total 51 patients with UTI had MCUG. Sixty-six percent were males and 82\% were below 2 years of age. VUR was found in $\mathbf{2 7 . 4 \%}$ cases and the highest number was in the age group of 2- 5 years. UTI in young children is largely overlooked and the observed frequency of VUR in children with UTI in our study is considerable, though slightly lower than reported. Therefore the standard protocol of performing MCUG as part of UTI work up in the high-risk group should be followed.

\section{Key Words: Urinary tract infections, Vesicoureteral reflux, Children.}

\section{INTRODUCTION}

Urinary tract infection (UTI) is a common bacterial infection in children. It is an important cause of acute illness in early childhood and has the potential to cause permanent renal damage if not treated timely especially in young children $(<2$ years). ${ }^{1,2}$ UTI in infants and young children may have few recognizable symptoms and signs other than fever and therefore can remain unrecognized for long periods of time. It is extremely important to identify those children who are at higher risk for renal damage. Besides, UTI serve as a marker for urinary tract abnormalities in children. ${ }^{1}$

The most commonly observed abnormalities in children with UTI is VUR which is seen in almost $30-40 \%$ children with UTI. ${ }^{3,4}$ Younger children are more likely to have reflux and reflux in younger children is more likely to resolve

* Patan Hospital, Patan, Nepal.

Address for correspondence : Dr. Shrijana Shrestha

Patan Hospital, Patan, Nepal.

Email: pathosp@healthnet.org.np spontaneously regardless of the grade of reflux. If resolution has to occur, it usually does so within the first few years after diagnosis. ${ }^{5}$ Children with VUR are at higher risk of reflux nephropathy and renal scaring. Children below two years are at higher risk of developing renal scarring. ${ }^{3}$ There is a direct relationship between grade of reflux and the incidence of nephropathy. Renal scarring can occur after only a single UTI, even in the absence of fever. ${ }^{5}$ Work ups to rule out VUR are therefore recommended after the first $\mathrm{UTI}^{3,5}$ and this abnormality is best detected and graded by MCUG. ${ }^{4,5}$

\section{OBJECTIVES}

1. To identify the presence of VUR in children with documented UTI

2. To analyze the relative frequency of its occurrence in relation to age and sex. 
MATERIAL \& METHODS

This was a retrospective hospital based study carried out in Department of Paediatrics, Patan Hospital during a 19month period between Jestha 2059 to Poush 2060 (15/6/ 2002-15/1/2004). All the children who underwent MCUG as part of UTI work up during the period were included in the study. The hospital numbers of the patients were traced from the $\mathrm{X}$ ray record book and the patients' files were studied and data analyzed.

\section{RESULTS}

During the study period a total number of 51 children underwent MCUG as part of UTI work up. The most common age group was less than two completed months (47\%) and $82 \%$ were below 2 years of age. Males were predominant $(66.6 \%)$ and the number of males was significantly high in the less than two-month age group.

Out of the 51 children 18 had more than one episode of documented culture proven UTI. Some of the urine cultures were done in outside hospitals/clinics. Those reports were excluded while analyzing the organisms isolated and antibiotic sensitivity pattern.

As a large proportion of our study group was less than 2 year olds, the mode of urine sample collection was mostly catheterization $(65.15 \%)$ followed by clean catch $(32.3 \%)$ and supra pubic puncture $(2.8 \%)$.

The in-hospital urine culture reports showed pure growth of single organism with significant colony counts in 63 samples. Similarly mixed growth of 2 organisms with significant colony counts were seen in 6 cases. Of these 6 cases 3 were catheter sample and 3 were clean catch. All the 6 cases were febrile sick babies with no other foci of infection and 2 of them had abnormal renal ultrasound. Repeat cultures on antibiotic were negative in five out of six cases.

Gram-negative organisms were isolated in $87.3 \%$ of all the positive urine cultures and the most common single organism isolated was E coli (71.4\%) followed by Klebsiella (12.6\%). Among the gram-positive organisms the most commonly isolated was coagulase negative Staphylococcus (coag-ve Staph) followed by coagulase positive Staphylococcus (coag +ve Staph).

The antibiotic sensitivity pattern of E coli showed that $60.8 \%$ of E coli were resistant to cotrimoxazole, $65.2 \%$ were resistant to amoxycillin and $58.6 \%$ were resistant to nalidixic acid. Overall $43.4 \%$ of E coli were resistant to all of the three most commonly used antibiotics (cotrimoxazole, amoxycillin and

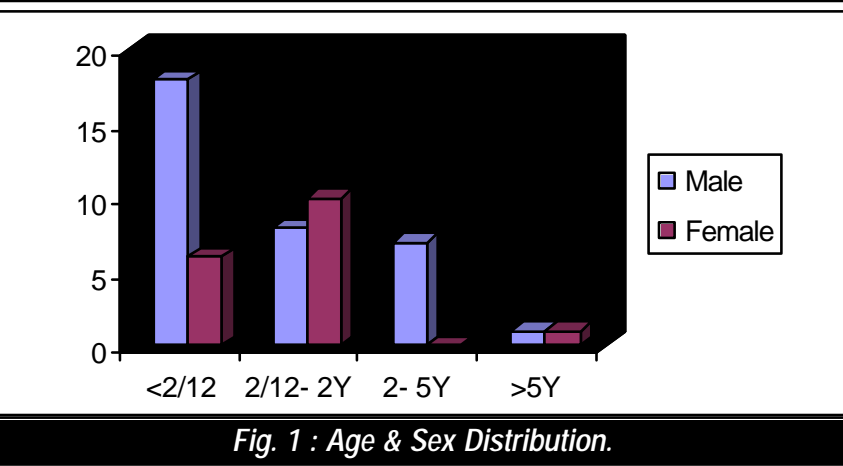

nalidixic acid) for the treatment of UTI.

Of all the gram negative organisms isolated in the urine culture specimen (single organism with significant colony counts) $52.8 \%$ were cotimoxazole resistant. (Cotrimoxazole sensitivity was not checked in 6 cases).

Urine microscopy reports were obtained in only 34 cases and $85 \%$ of them had more than 5 white blood cells per high power field.

Ultrasound (USG) report was abnormal in $26(50.9 \%)$ cases and the most common USG findings were unilateral hydronephrosis (26.9\%) and bilateral enlarged echogenic kidneys $(26.9 \%)$. Interestingly all of the unilateral hydronephrosis were left sided. Among those with hydronephrosis, $63 \%$ were reported mild hydronephrosis while mild to moderate and moderate were $18 \%$ each. One of the moderate hydronephrosis on follow-up USG showed gross hydronephrosis.

Fourteen cases (27.4\%) showed positive VUR on MCUG and the percentage of VUR among less than 1 year old were only $13 \%$. Seven out of fourteen (50\%) were of the age group 2 to 5 years. Among the VUR positive cases boys were $57.1 \%$ as two third of the study population were males. However the incidence of VUR was more in females (35.2\%) compared to males $(23.3 \%)$.

Seven cases had unilateral and seven had bilateral VUR. One boy with VUR also had posterior urethral valve, which was corrected but after that patient lost follow up.

Of the VUR positive cases, $35.7 \%$ had more than two episodes of documented culture positive UTI while $21 \%$ had only one documented culture positive UTI before MCUG. Six out of fourteen cases with VUR (42.8\%) had normal USG; one case each of grade I \& grade IV VUR and two cases each of grade II \& grade III VUR.

Three cases had breakthrough infection on cotrimoxazole prophylaxis and two cases had UTI on prophylaxis defaulter. 
Table I : Organisms isolated in catheter sample

(Pure growth of single organism with significant colony count)

\begin{tabular}{lc|cc}
\hline \multicolumn{1}{c}{ Organisms } & $\mathbf{> 1 0}^{\mathbf{5}}$ colonies & $\mathbf{> 1 0}^{\mathbf{4}}$ colonies & $\mathbf{> 1 0}^{\mathbf{3}}$ colonies \\
\hline E coli & 19 & 6 & $2^{*}$ \\
\hline Klebsiella & 5 & 1 & \\
\hline Proteus & 2 & & \\
\hline Coag + ve Staph & 1 & & \\
\hline Coag -ve Staph & 2 & 1 & \\
\hline Non haem Step & 1 & & \\
\hline Enterobacter & 2 & & \\
\hline
\end{tabular}

* Both had abnormal USG

\# Sick 5 day old neonate with no other foci of infection

Table II : Organisms isolated in clean catch sample (Pure growth of single organism with significant colony count)

\begin{tabular}{l|c|c}
\hline \multicolumn{1}{c|}{ Organisms } & $\mathbf{> 1 0 ^ { 5 } \text { colonies }}$ & $>\mathbf{1 0}^{4}$ colonies \\
\hline E coli & 15 & $1+$ \\
\hline Klebsiella & 2 & \\
\hline On antibiotics from outside
\end{tabular}

Table III : Organisms isolated in SPA sample (Pure growth of single organism with significant colony count)

\begin{tabular}{c|c|c}
\hline Organisms & $\mathbf{> 1 0}^{\mathbf{5}}$ colonies & $>\mathbf{1 0} \mathbf{4}$ colonies \\
\hline E coli & 1 & 1
\end{tabular}

These breakthrough infections were treated and were restarted on prophylaxis.

A seven-year-old girl with bilateral grade II reflux had hypertension and progressively rising serum creatinine. A three-month-old girl with bilateral grade $\mathrm{V}$ reflux and a sixmonth-old girl with bilateral grade II reflux had elevated serum creatinine for age. None of the others had hypertension or impaired renal function/ elevated serum creatinine.

Three cases underwent corrective surgery for VUR, two in our hospital and one at India. Of those who underwent surgery, one was a three and a half year old girl with left sided grade IV VUR with recurrent UTI and failure to thrive. The second case was a four-year-old boy with left sided grade III VUR with recurrent febrile UTIs on prophylaxis and failure to thrive. He also had recurrent seizures precipitated by fever (multiple neurocysticercosis on CT scan), requiring repeated hospital admissions. The third one was a two and a half year old boy with right grade II VUR who also had recurrent episodes of

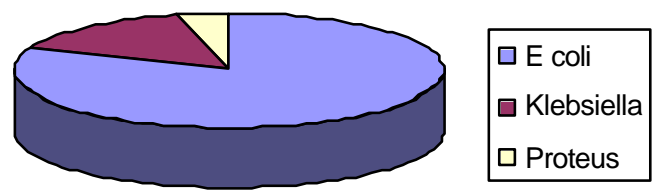

Fig. 2 : Gram -ve organisms isolated. (Single organism with significant colony count) supraventricular tachycardia and gone to India for its evaluation where he underwent corrective surgery for VUR. One child who underwent surgery for correction of VUR had persistence of reflux post surgery shown on repeat MCUG after eight month of surgery when he presented with another episode of febrile UTI.

Three more cases were referred for surgery and they were: (i) a seven year old girl with bilateral grade II VUR, hypertension and progressively rising serum creatinine, (ii) a three month old girl with bilateral grade IV VUR and slightly elevated serum creatinine for age and (iii) a two year old boy with bilateral grade IV VUR.

Two cases lost to our follow up and those who underwent surgery for VUR or were referred for surgery also did not come for follow up visits to us except for the one child with persistence of VUR post surgery. The remaining seven cases are in regular follow up.

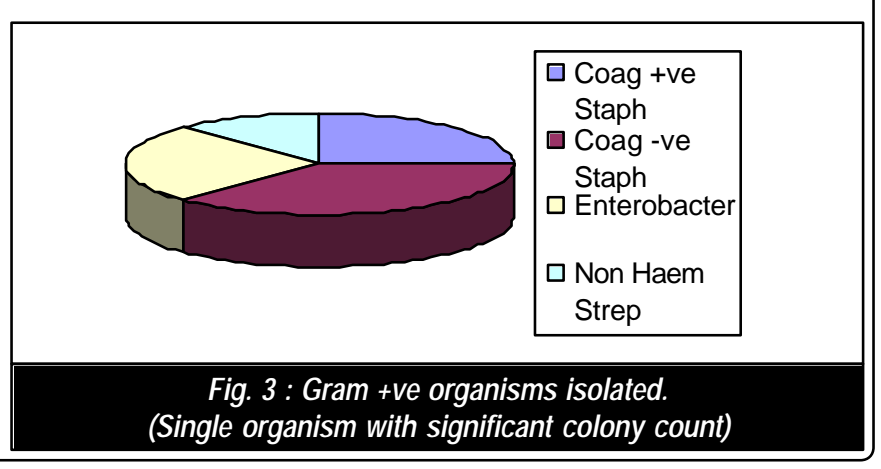


Table IV : In-hospital resistance pattern of $\mathbf{E}$ coli

\begin{tabular}{l|c}
\multicolumn{1}{c|}{ Drugs resistance } & Percentage \\
\hline Cotrimoxazole & $60.8 \%$ \\
\hline Amoxycillin & $65.2 \%$ \\
\hline Nalidixic Acid & $58.6 \%$ \\
\hline Ciprofloxacin & 19.55 \\
\hline Cefotaxim & $17.3 \%$ \\
\hline Gentamycin & $13 \%$ \\
\hline
\end{tabular}

Table V : Abnormal ultrasound findings

\begin{tabular}{l|c}
\hline \multicolumn{1}{c|}{ Ultrasound findings } & No. of cases \\
\hline Bilateral enlarged echogenic kidneys & $7(26.9 \%)$ \\
\hline Unilateral hydronephrosis & $7(26.9 \%)$ \\
\hline Cystitis & $6(23 \%)$ \\
\hline Unilateral hydronephrosis + hydroureter & $2(7.69 \%)$ \\
\hline Unilateral hydronephrosis + cystitis & $2(7.69 \%)$ \\
\hline Unilateral enlarged echogenic kidney & $1(3.8 \%)$ \\
\hline Unilateral small kidney & $1(3.8 \%)$ \\
\hline
\end{tabular}

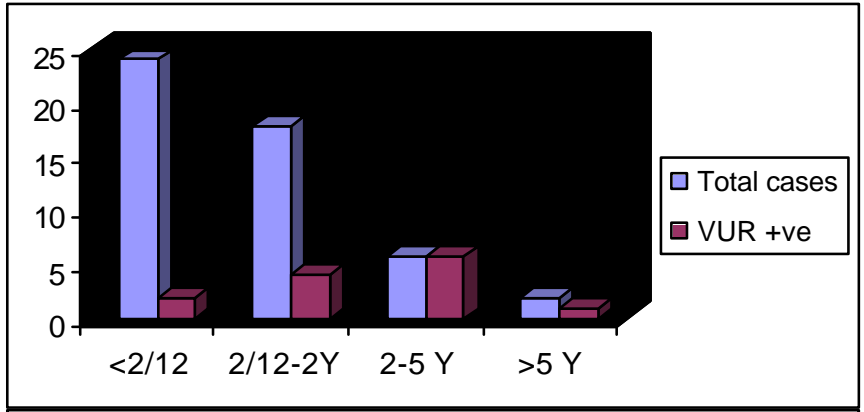

Fig. 4 : VUR in relation to age group.

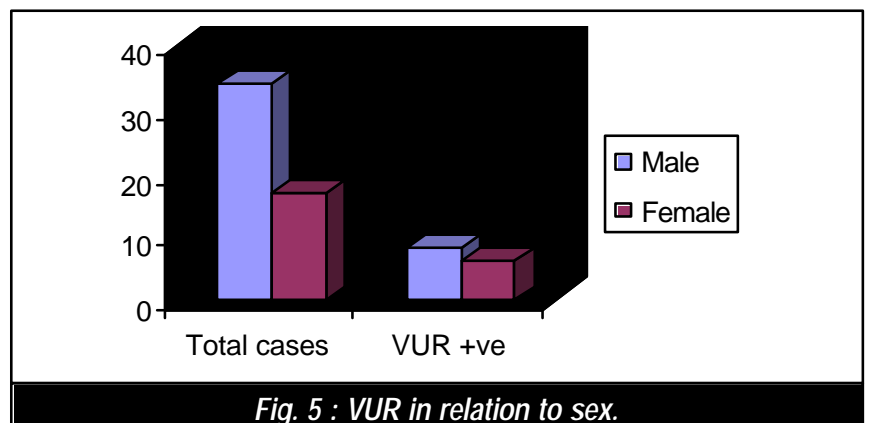

Among the regular follow up cases one nine month old girl with left sided grade III reflux showed poor weight gain and failure to thrive (has a normal DMSA scan) while the rest were growing fine.

\section{DISCUSSION}

During the first year of life UTI is more common in boys with the male female ratio of 2.8-5.4:1. ${ }^{4}$ We in our study observed the male female ratio of $2: 1$ in children less than one year. Males are highly susceptible to UTIs below three month of age $^{1}$ and our figure of significantly higher number of males below two months of age, also tallies with this.
It is reported that beyond 1-2 years of age there is striking female preponderence. ${ }^{4}$ However in our study, except for the age group of two months to two years female preponderance was not noted in any other age groups and two third of our study group were males. One explanation could be the possible less attention to a female child's illness and less frequent chances of bringing to the hospital or any medical service. As almost half of the study group was the children below two completed years, clean catch mid stream urine collection not being a reliable method in these children, catheterization was our most common mode of urine collection and SPA was used less frequently for the purpose. American academy of Paediatrics, subcommittee on UTI recommends that urine obtained by catheterization or SPA is unlikely to be contaminated, hence either of these modes of sample collection should be used for establishing the diagnosis of UTI. ${ }^{1}$

E coli is the most common pathogen causing $\mathrm{UTI}^{6,7}$ and organisms other than $\mathrm{E}$ coli are encountered with higher frequency among children with recurrent UTIs. ${ }^{7}$ In our study

E coli was the most common pathogen causing UTI and among the gram-positives, coag -ve Staph was top on the list. Staphylococcus and Enterobacter are described as the most common gram-positive organisms ${ }^{8}$ while coag -ve Staph is now being recognized more as a urinary pathogen in children ${ }^{7}$

In our study VUR was detected in $27.4 \%$ of children with documented UTI and among less than one year olds only $13 \%$ showed VUR. Incidence of VUR in children with UTI varies between 30 to $50 \%$ and in a study by Royal college of Paediatrics and Child Health Research Unit (RCPHC RU) done in 2001 the reported incidence is 36\%.6 Reports have shown that the rate of VUR among children less than one year with 
UTI exceeds $50 \%{ }^{5}$ but we found only $13 \%$ of our less than one year old with UTI had VUR for reasons not clear. One possible reason for the slightly less overall incidence of VUR in our study and especially the lower figure in the less than one year olds could be that we in our hospital are not using Fluroscopy for MCUG and that a small number of cases might have been be missed during the routine MCUG. The average age at diagnosis of VUR is two to three years ${ }^{4}$ and $50 \%$ of our children with VUR were of the age group two to five years. VUR is more common in females except in the neonatal age group. Of all the VUR detected during UTI work up, $80 \%$ are females. ${ }^{4}$ We in our study found VUR slightly more in females with UTI (35.2\%) than in males (23.5\%). We had normal USG report in $42.8 \%$ cases with VUR. USG is insensitive in detecting reflux, especially the lower grades of reflux and only $40 \%$ of children with reflux have any abnormality in USG. ${ }^{4,5}$ Among the children with VUR in our study, 21\% had only one episode of documented UTI before MCUG. It is recommended than children should be investigated for VUR at the first documented UTI as they are very likely to have another. ${ }^{5,9}$ and according to the 'big bang' theory proposed by Ransley and Risdon (1978) the most severe degree of renal parenchymal injury occurs with the first infection. ${ }^{5}$

The natural history of VUR varies with the grade of reflux and age at the time of diagnosis. With high grade VUR renal scaring is 4-6 times more likely than with low grade VUR. ${ }^{1}$ Treatment options for VUR are continuous antibiotic prophylaxis or surgical repair. International reflux study observed spontaneous resolution of VUR in $25 \%$ cases. Of the spontaneous resolutions $90 \%$ occur within first 5 years and the duration is shorter if the reflux is diagnosed before one year of age. ${ }^{9}$ Therapeutic recommendations therefore depend on the age of the patient, grade of VUR, renal scarring and occurrence of breakthrough infections., ${ }^{2,45}$ As we were not doing renal scans routinely due the cost factor, renal scarring was not used as one of the marker for choosing the therapeutic options. Recurrent breakthrough infection on antibiotic prophylaxis was used as one of the clinical tool for decisionmaking. The rate of breakthrough infections on prophylaxis was not high and none of our cases required change of prophylactic antibiotic or double prophylaxis. It has been stated that following breakthrough infection change of prophylactic antibiotic is usually not required. ${ }^{2}$ One child had persistence of VUR post surgery. The success rate of surgery in primary VUR is very high and other than technical errors the reason for failure could be inability to identify voiding dysfunctions and secondary reflux. ${ }^{5}$

\section{CONCLUSIONS AND RECOMMENDATIONS}

UTI in infant and young children are greatly overlooked and it has the potential to cause renal damage, any child less than two years with febrile illness without a clear foci, should be investigated for UTI. Looking at the positive yield of MCUG in children with documented UTI, The standard guidelines for performing MCUG as part of UTI work up in children below five years with the documented UTI should be followed and normal USG should not be considered as an indication of not performing MCUG in those who need it.

With the given in hospital sensitivity reports, cotrimoxazole or amoxycillin should not be used for treatment of definite UTIs unless the urine culture reports are favorable. Nalidixic acid should not be used for the treatment of febrile UTI in children, as it doesn't reach adequate serum level needed to treat pyelonephritis. In spite of high cotrimoxazole resistance rate among gram-negative organisms, especially E coli, the rate of breakthrough infections on prophylaxis is relatively low. Corimoxazole still can be continued as the drug of choice for UTI prophylaxis in children above six weeks of age.

Due to the inheritance pattern of VUR (approximately 35\% of the sibling with VUR and 50\% of offspring of mother with VUR also has reflux ${ }^{4,5}$ ) we should also start screening these at risk children.

In view of the small sample size, no clear inferences could be drawn from the study regarding the age and sex distribution of VUR. A larger prospective study with bigger sample size and a longer follow up period is required.

\section{REFERENCES}

1. Practice parameter: The diagnosis treatment and evaluation of the initial UII in febrile infants and young children. Pediatr 1999; 103:843-852.

2. Consensus statement on management of UTIs. Indian Pediatr 2001; 38:1106-1115.

3. Management of urinary tract infections. Ind J Pediatr2003; $70: 235-239$.

4. Nelsons textbook of Paediatrics, 17th ed. 2004.

5. Campbell's Urology, 7th ed. 1998.

6. Dignosis and management of UIIs in children under two years. Assessment of practice under published guidelines. Royal college of Paediatrics and Child health Research Unit. March 2001 .

7. Hellerstein S. Urinary tract infections. Pediatr Clin North Am 1995; 42: 1433-1457.

8. Roshton HG. Urinary tract infections in children: Epidemiology, evaluation and management. Pediatr Clin North Am. 1997; $44: 1133-1169$.

9. Belman BA. Vesicoureteral reflux. Pediatr Clin North Am1997; $44: 1171-1190$. 